\title{
Lattice Radiotherapy with RapidArc for Treatment of Gynecological Tumors: Dosimetric and Early Clinical Evaluations
}

Beatriz E. Amendola ${ }^{1}$, Naipy Perez ${ }^{2}$, Marco a. Amendola ${ }^{2}$, Xiaodong $\mathrm{Wu}^{3}$, Mansoor M. Ahmed $^{4}$, Alejandro J. Iglesias ${ }^{1}$, Richard Estape ${ }^{5}$, Nicholas Lambrou ${ }^{5}$, Pietro Bortoletto 6

1. Innovative Cancer Institute 2. Innovative Cancer Institute, 6141 Sunset Drive, South Miami, FL 33143

3. CyberKnife Center of Miami; University of Miami Miller School of Medicine, Miami, FL 4. Department of Radiation Oncology, Miami University 5. South Miami Gynecologic Oncology Associates 6. Feinberg School of Medicine

$\square$ Corresponding author: Beatriz E. Amendola, dramendola@gmail.com Disclosures can be found in Additional Information at the end of the article

\section{Abstract}

Background: We report the case of a patient with an advanced carcinoma of the cervix treated using the new concept of LATTICE Radiotherapy (LRT). We describe the dose distribution, planning and delivery of treatment as well as the clinical evaluation of the patient during and at the completion of treatment. The basic principle of LATTICE is to create, within the tumor volume, multiple localized high-dose islands with a certain degree of separation to form lower dose regions.

Methods: This is case report based on a 48-year-old female, G1P1, who presented with advanced cervical carcinoma and a bulky pelvic mass. The patient was treated with Trilogy equipment using IMRT. She received two fractions of $3 \mathrm{~Gy}$ each to the pelvis, on an emergent basis, using conventional 3-D conformal irradiation. Following this course, she was treated with LATTICE radiotherapy (LRT) in order to create a non-homogeneous dose distribution analogous to interstitial brachytherapy, combined with conventional IMRT.

Results: The patient underwent TAH/BSO six weeks post-LATTICE and there was complete clinical pathological response. She continues with maintenance chemotherapy, and at this time the patient is NED.

Conclusions: LATTICE Radiotherapy (LRT) using Rapid Arc appears to be a safe and effective approach for the treatment of bulky gynecological tumors. Further prospective clinical trials need to be developed in order to test this hypothesis.

Published 09/27/2010

(c) Copyright 2010

Amendola et al. This is an open access article distributed under the terms of the Creative Commons Attribution License CC-BY 3.0., which permits unrestricted use, distribution, and reproduction in any medium, provided the original author and source are credited.
Categories: Obstetrics/Gynecology, Radiation Oncology, Oncology

Keywords: chemotherapy, gynecological tumors, imrt, lattice radiotherapy, rapidarc, cervical cancer

\section{Introduction}

The concept of 3D LATTICE Radiotherapy (LRT) has been recently described by Wu, et al. [1-2]. Its basic principle is to create within the tumor volume multiple localized high-dose islands with a certain degree of separation to form lower dose regions. This new proposal for radiation therapy delivery is based on: 1) the proven effectiveness of boosting tumor response using 2D Spatial Fractionated GRID Radiotherapy (SFGRT), and 2) the new advances in radiation delivery

\section{How to cite this article}

Amendola B E, Perez N, Amendola M a., et al. (September 27, 2010) Lattice Radiotherapy with RapidArc for Treatment of Gynecological Tumors: Dosimetric and Early Clinical Evaluations. Cureus 2(9): e15. DOI 10.7759 /cureus. 15 
which allow creating three dimensional (3D) high dose regions inside the tumor volume without the limitation of high toxicity in the peripheral normal tissue regions [3-5].

Dose escalation for gynecological tumors using field within a field Intensity Modulated Radiation Therapy (IMRT) is a technique that has been used and reported by Mundt [6] with favorable results. LTR is a different approach which pursuits not only the dose escalation but also the idea of facilitating or promoting intra-tumoral bystander effect, thus increasing the biological effectiveness of the treatment. Published data reviewed by Peters, et al. [7] strongly suggest that GRID therapy induces a rapid and higher rate of tumor cell apoptosis in bulky and hypoxic tumors. This technique is very appealing to treat patients with voluminous gynecological tumors.

We report a case of a patient with a bulky squamous cell carcinoma of the uterine cervix treated with this approach. The main goal was to create concurrently multiple highly focused high dose regions inside the bulky tumor to increase the response of the tumoral cells through an anticipated localized bystander effect and improve the local control.

\section{Case Presentation}

A 48-year-old female, G1P1, presented to the emergency room with a history of low pelvic pain, persistent severe vaginal bleeding and anemia. Upon admission, she was found to have a large indurated mass in the uterine cervix. Emergent surgical exploration with pelvic and para-aortic lymphadenectomy revealed a Stage IIB squamous cell carcinoma of the cervix with metastases to the right obturator and internal iliac lymph nodes. Due to the large size of the cervical tumor with an estimated volume of about $250 \mathrm{cc}$ and the profuse bleeding, hysterectomy was not performed and the patient was referred to radiation oncology for further management. Due to persistent bleeding which required transfusion, the patient initially received two fractions of 3 Gy each to the pelvis, on an emergent basis, using conventional 3-D conformal irradiation. She was also placed on concomitant chemotherapy consisting of six consecutive weekly cycles of Cisplatin $(40 \mathrm{mg} / \mathrm{m} 2)$. It was then decided to treat the patient with LATTICE Radiotherapy in order to create a non-homogeneous dose distribution with the purpose of creating a faster response and debulk the tumor prior to surgery.

The planning target volume (PTV) was obtained by placing a $5 \mathrm{~mm}$ margin around the clinical target volume (CTV, $915 \mathrm{cc}$ ) which included the uterine cervix mass $(250 \mathrm{cc})$ and the regional retroperitoneal and pelvic lymph nodes. In addition, fifteen small spheres of $1 \mathrm{~cm}$ in diameter were created inside the gross tumor volume (GTV) to deliver high dose LRT that we denominated as High Dose Gross Tumor Volume (HDGTV) as seen in Figure 1 and Figure 2. 


\section{Cureus}

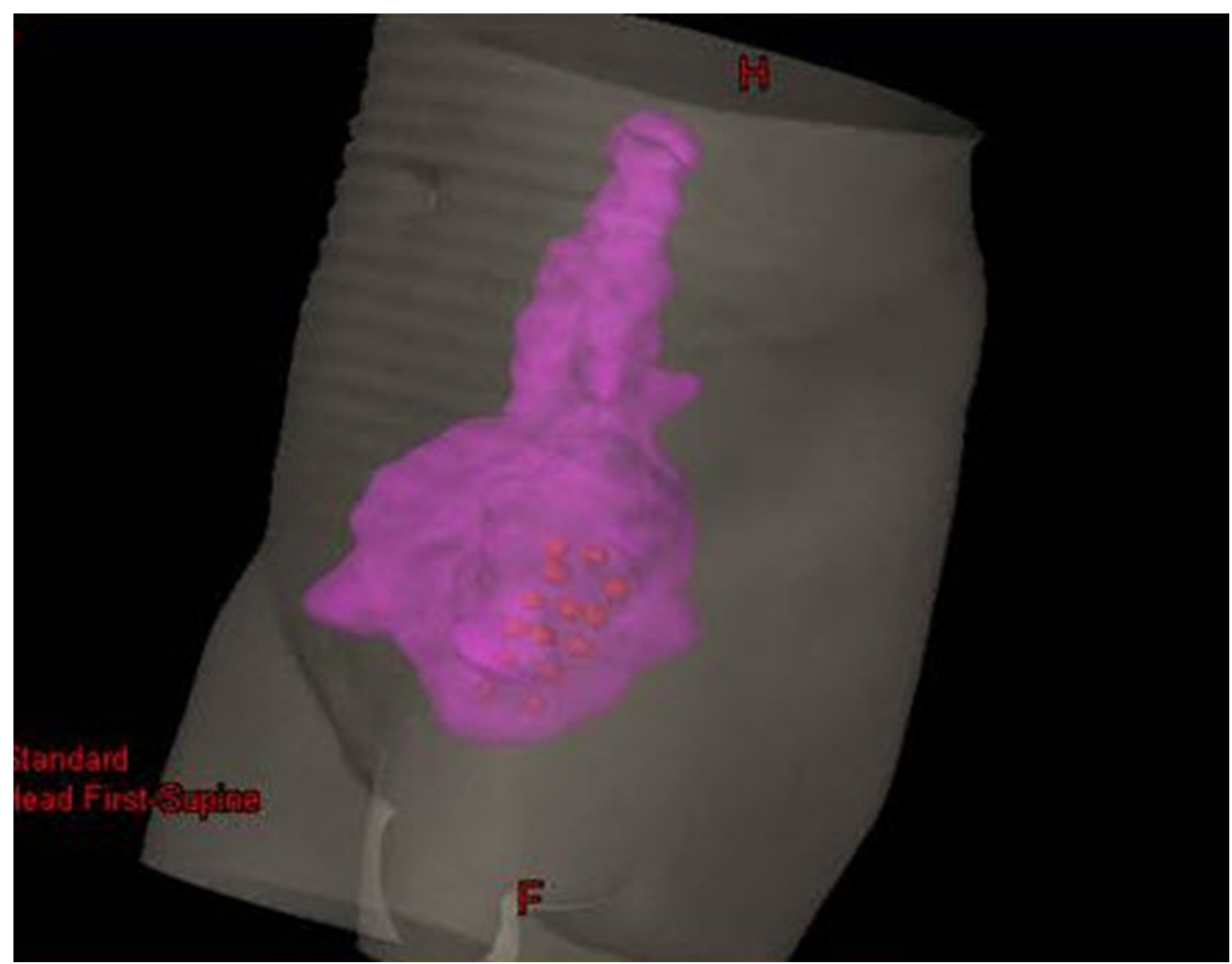

FIGURE 1: Isodose rendering of the target volume treated including high and low doses of radiation therapy

Coronal CT reconstruction with 3D rendering PTV in pink showing areas of higher dose (HDGTV) in red.

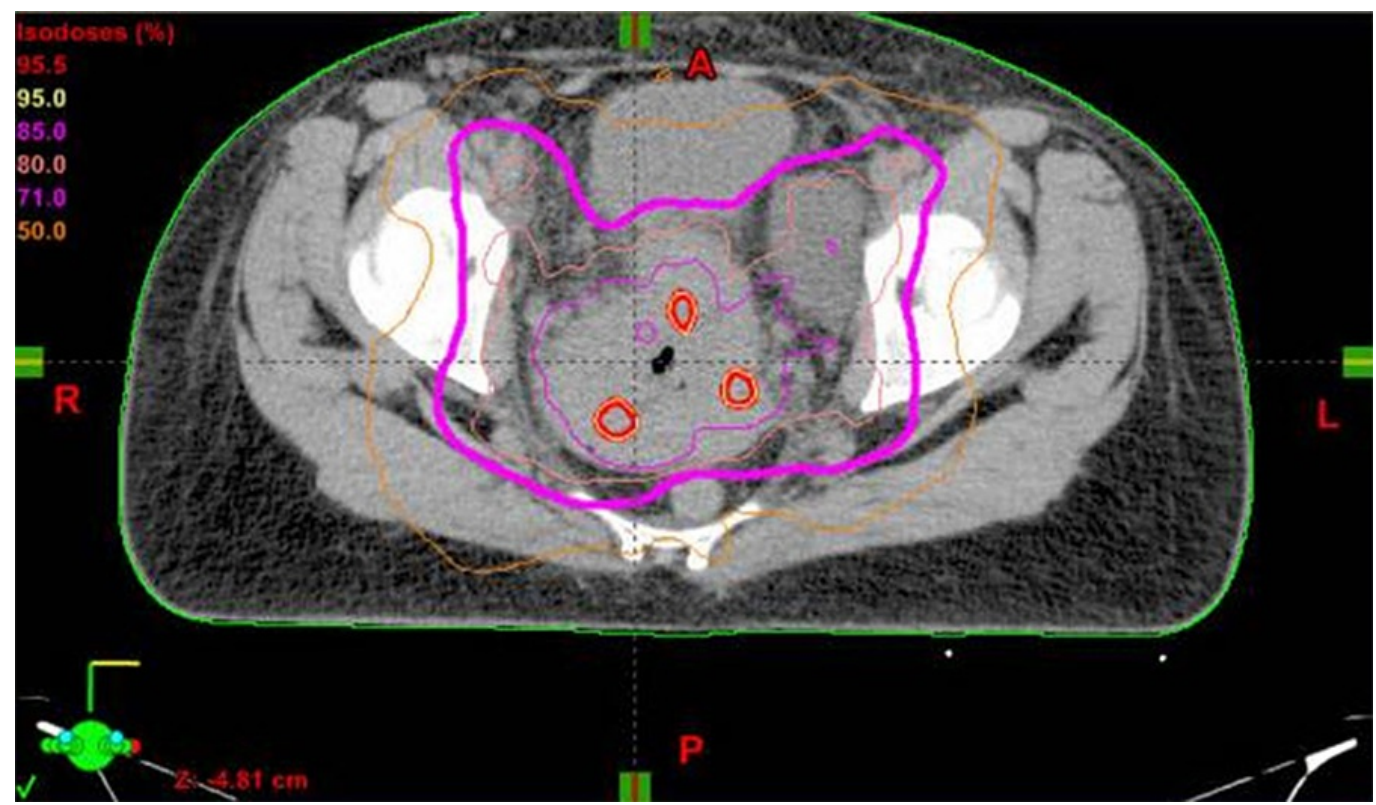

FIGURE 2: Isodose distribution of the initial volume

Axial CT of the mid-pelvis through the tumor mass with the isodose distribution showing the regions of dose escalation using the LATTICE technique. 


\section{Cureus}

The average distance between these high dose spheres (center to center) was $3 \mathrm{~cm}$.

Dose planning was performed using the Eclipse Planning System ${ }^{\mathrm{TM}}$. A volumetric arc (RapidArcTM) technique using two complete $6 \mathrm{MV}$ arcs was employed to deliver 20 fractions of 1.8 Gy each to the PTV with a Trilogy linear accelerator (Varian Medical Systems, Palo Alto, CA). The HDGTV was planned to receive an escalated dose of $2.4 \mathrm{~Gy}$ per fraction, concurrently using the same two arcs. The LRT plan was prescribed as follows: PTV received $180 \mathrm{cGy}$ per fraction (3600 cGy) and the 15 spheres GTV-HD received 240 cGy per fraction (4800 cGy)

Evaluation of the patient at completion of LRT treatment after 22 daily fractions over 32 elapsed days demonstrated an excellent clinical symptomatic response with cessation of vaginal bleeding and absence of a palpable mass on pelvic exam. She was then reassessed with CT of the abdomen and pelvis, which demonstrated marked reduction of the tumor volume from 250 cc to 75 cc (70\% reduction) as seen in Figure 3.

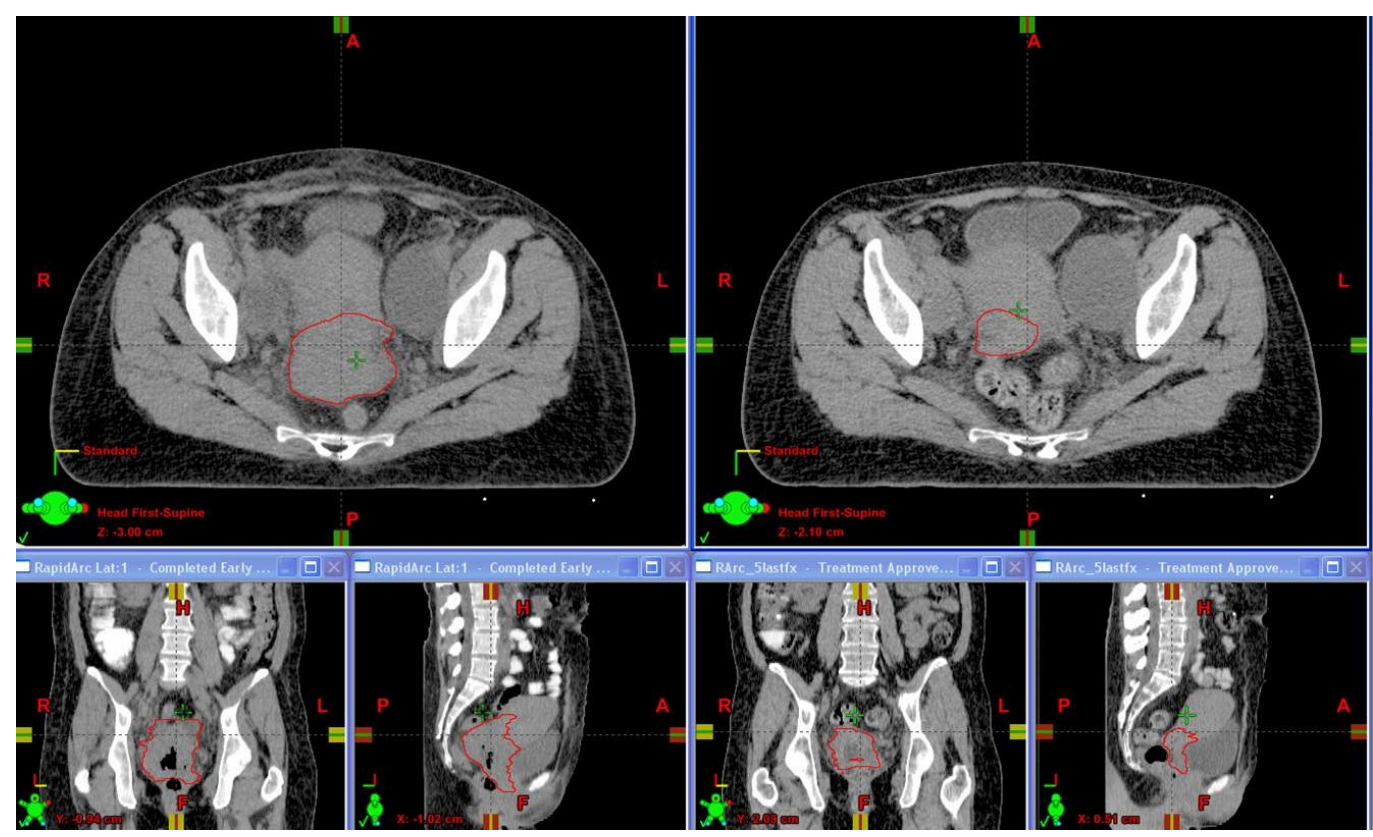

\section{FIGURE 3: LATTICE radiation treatment showing marked reduction in the tumor volume}

CT of the pelvis in axial, coronal and sagittal planes before (left) and after (right) LATTICE radiation treatment showing marked reduction in the tumor volume.

Using this CT, a new plan was created for a final boost to deliver five additional fractions using conventional IMRT with $1.8 \mathrm{~Gy}$ per daily fraction to the residual mass. The total dose received by the PTV was $51 \mathrm{~Gy}$ (BED10 of $61 \mathrm{~Gy}$ ), while the HDGTV received $63 \mathrm{~Gy}$ (BED10 of $78 \mathrm{~Gy}$ ). The patient underwent total abdominal hysterectomy and bilateral salpingo oophorectomy (TAH/BSO) six weeks post LATTICE Radiotherapy showing a complete clinical pathological response. She continued with maintenance chemotherapy, and at the time of this writing the patient is without evidence of disease (NED). The only acute effect probably related to radiation treatment was diarrhea, easily controlled with a diet and oral medication.

\section{Discussion}


The introduction of the new concept of LATTICE Radiotherapy (LRT) has the potential of improving outcomes in the treatment of bulky tumors with radiation therapy (1-2). We are reporting a case of a woman with a voluminous squamous cell carcinoma of the uterine cervix treated using an array of high dose spheres inside the very large mass. The periphery of the tumor together with the rest of the clinical target volume received a conventional dose fractionation.

The implementation of combined chemo-radiation and IMRT, ten years ago, represented a substantial progress in the management of locally advanced cervical cancer [8]. Radiationinduced bystander effects may open a new paradigm for future progress. As reviewed by Peters, et al. [7], high-dose radiation given through GRID is known to induce cytokines, such as TNF- $\alpha$ as well as ceramide, a potent inducer of apoptosis in endothelial cells. Presence of these factors released from tumor cells that are directly hit by high-dose LRT provides a platform to sensitize the effects of subsequent fractionated radiation given after LRT.

The 3D dose lattice can be generated with single or multiple arcs, with a dynamic Multileaf Collimator (MLC) gantry linear accelerator and/or non-coplanar focused beams to form a highly convergent dose distribution, such as the ones used for stereotactic radiosurgery (SRS) or stereotactic body radiotherapy (SBRT) [1-2, 9]. This makes LATTICE Radiotherapy a practically achievable alternative to traditional GRID therapy, which delivers a highly heterogeneous dose distribution, anticipated to trigger the bystander effect of radiation [7]. With high dose regions strictly contained within the target volume, normal tissue toxicity is practically avoided providing an increased therapeutic ratio. This case report illustrates that it is technically feasible to create higher dose regions inside the tumor mass without increasing the risk of early toxicity using arc therapy and thereby resulting in a dramatic tumor response.

It should be noted that in the reported case, the LRT was administered concurrently with the conventional fractionation treatment, and the higher dose delivered to the volume of interest was less than the high dose range of traditional SFGRT. Therefore, rather than a robust bystander effect, a cumulated bystander effect may have been elicited by LRT throughout the treatment.

It must be noted that, in the U.S., patients with IIB cervical cancer are generally treated with radiation alone. However, in this particular young patient, with a very large, irregular, cervical tumor mass, it was deemed that post-radiation hysterectomy with curative intent was needed to increase the probability of disease-free survival.

There are reports in the literature as the recent article by Huguet, et al. [10] that show that in bulky early-stage cervical carcinoma ( $>4 \mathrm{~cm}$ in diameter) there is an increased incidence of treatment failure using radiation alone. This has been also the experience of the Villejuif Institute [11], where patients did better when treated with radiation therapy combined with concurrent chemotherapy, followed by hysterectomy.

A limitation of this approach is that this technique is not feasible with many currently available radiation therapy units. It requires daily monitoring with image guidance with a high degree of precision to prevent high doses being delivered to normal tissue structures.

Acute toxicity was very limited and not more than what is usually experienced in similar cases.

\section{Conclusions}

In this preliminary experience with a single case, LATTICE Radiotherapy using RapidArcTM, appears to be an effective approach for the treatment of bulky gynecological tumors. Further 
prospective clinical trials need to be developed in order to test this hypothesis.

\section{Additional Information \\ Disclosures}

Human subjects: Consent was obtained by all participants in this study. Conflicts of interest: In compliance with the ICMJE uniform disclosure form, all authors declare the following:

Payment/services info: All authors have declared that no financial support was received from any organization for the submitted work. Financial relationships: All authors have declared that they have no financial relationships at present or within the previous three years with any organizations that might have an interest in the submitted work. Other relationships: All authors have declared that there are no other relationships or activities that could appear to have influenced the submitted work.

\section{Acknowledgements}

Authors' contributions: Dr. B. Amendola and Dr. M. Amendola contributed with the preparation and editing of the manuscript. Dr. Wu, Ms. Perez, and Mr. Iglesias contributed with the Physics and Dosimetry. Dr. Estape and Dr. Lambrou contributed with the surgical reports and clinical / surgical follow-ups. Dr. Ahmed contributed with the Radiobiological aspect of this paper. Mr. Bortoletto is a medical student that worked with us on the project. Acknowledgements : Christina Cascante

\section{References}

1. Wu X, Ahmed M, Pollack A: On modern technical approaches of 3D high-dose Lattice radiotherapy (LRT). Int J Radiat Oncol Biol Phys. 2009, 75:723.

2. Wu X, Ahmed M, Wright J, Gupta S, Pollack A: On modern technical approaches of threedimensional high dose Lattice radiotherapy (LRT). PeerEMed.com. 2010, ID 19353:Accessed: March 5, 2010: peeremed.com.

3. Mohiuddin M, Fujita M, Regine W, et al.: High-dose spatially fractionated radiation (GRID) : A new paradigm in the management of advanced cancer. Int J Radiat Oncol Biol Phys. 1999, 45:721-727.

4. Zwiker R, Meigooni A, Mohiuddin M: Therapeutic advantage of GRID irradiation for large single fractions. Int J Radiat Oncol Biol Phys. 2004, 58:1309-1315.

5. Neuner G, Vander Walde N, Ha J, et al.: High-dose spatially- fractionated GRID radiation therapy (SFGRT): A comparison of outcomes of treatment delivered through Cerrobend GRID versus MLC GRID. Int J Radiat Oncol Biol Phys. 2008, 72:488. 10.1016/j.ijrobp.2011.01.065

6. Mundt A, Lujan A, Rotmensch J, et al.: Intensity modulated whole pelvic radiation therapy in women with gynecologic malignancies. Int J Radiat Oncol Biol Phys. 2002, 52:1330-1337.

7. Peters M, Shareef M, Gupta S, et al.: Potential utilization of Bystander/ Abscopal- Mediated signal transduction events in the treatment of solid tumors. Current Signal Transduction Therapy. 2007, 2:129-143.

8. Tanderup K, Georg D, Potter R, et al.: Adaptive Management of Cervical Cancer Radiotherapy . 2010, 20:121-129. 10.1016/j.semradonc.2009.11.006

9. Brown W, Wu X, Amendola B, et al.: Treatment of early non-small cell lung cancer, Stage IA, by image guided robotic stereotactic radioablation-Cyberknife. The Cancer Journal. 2007, 13:87-94.

10. Huguet F, Cojocariu O, Levy P, et al.: Preoperative Concurrent Radiation Therapy and Chemotherapy for Bulky Stage IB2, IIA and IIB Carcinoma of the Uterine Cervix with Proximal Parametrial Invasion. Int. J. Radiation Oncology Biol. Phys.. 2008, 72:1508-1515. doi: 10.1016/j.ijrobp.2008.03.054

11. Gerbaulet A, Kunkler I, Kerr G, et al.: Combined radiotherapy and surgery: Local control and complications in early carcinoma of the uterine cervix - the Villejuif experience, 1975-1984. Radiother Oncol. 1992, 23:66-73. 